\title{
Las políticas culturales en la industria cine- matográfica: la producción y exhibición de cine documental en Argentina (200I-2015)
}

\author{
Alejandra Cecilia Carril \\ Universidad Nacional del Litoral (UNL, Argentina).
}

\section{Resumen}

El presente trabajo pretende dar cuenta de la política cultural impulsada en nuestro país a principios del XXI en relación a la industria cinematográfica. Interesa indagar un ámbito generalmente menospreciado en el presupuesto estatal, o al menos no tenido en cuenta al mismo nivel que la producción, circulación y difusión del cine de ficción: el campo del cine documental. Se trata de un campo que cobra relevancia en Argentina a partir de 2001, cuando a la producción de algunos realizadores que se venía desarrollando en la transición democrática, se suma la de numerosos colectivos de realizadores en la coyuntura de crisis política, social y económica que se gesta con el recrudecimiento del neoliberalismo y en el marco de cambios estructurales en el campo de la tecnología que dan lugar a nuevas formas de producción y distribución del material audiovisual. Las acciones político-militantes de las asociaciones
Palabras clave políticas culturales, cine documental 
representativas del sector -la mayoría constituidas entre fines de los '90 y principios de los 2000- se traducirán en el período abordado en un reconocimiento y trato especial por parte del Instituto Nacional de Cine y Artes Audiovisuales para la producción documental, generándose una estrategia de promoción y subvención. En primer lugar se recorren algunas particularidades de la industria cinematográfica argentina, para explicar la alta concentración económica y geográfica que la caracteriza y la imperiosa necesidad de apoyo estatal para sostener la actividad cinematográfica en el tiempo. En segundo lugar se indaga en las políticas sostenidas en las últimas décadas desde el Estado argentino en el sector cinematográfico, que han marcado un desfasaje entre la producción y la comercialización. En tercer lugar se analizan los cambios producidos en la industria cinematográfica argentina y latinoamericana a partir de 2008 con el avance de la digitalización, que si bien representa en lo inmediato una mejora en las condiciones de exhibición, tiene a mediano y largo plazo consecuencias que acentúan la concentración oligopólica. En cuarto lugar se aborda el campo de la producción de cine documental, dando cuenta de las formas de trabajo y los circuitos de exhibición de los grupos de realizadores, que se consolidan en el marco de la crisis argentina de 2001 y los cambios que supone la tecnología digital. En quinto lugar se examinan los avances logrados en el período en función de las demandas de las asociaciones de documentalistas a través del Plan de Fomento del Instituto Nacional de Cine y Artes Audiovisuales, así como las cuentas pendientes sobre todo en materia de distribución y exhibición de cine documental. 
Abstract

Cultural Policies in the cinematographic industry: production and exhibition of documentary films in Argentina (2001-2015)

The present work aims to account for the cultural policy promoted in our country at the beginning of the $2 \mathrm{I}^{\mathrm{st}}$ century in relation to the film industry. We intend to research a generally underrated area in the state budget, or at least not taken into account at the same level as the production, circulation and diffusion of fiction films: the field of documentary cinematography. It is a field that becomes relevant in Argentina in the year 200I, when the production of some filmmakers that had been developing in the democratic transition, adds to that of many groups of filmmakers in the context of political, social and economic crisis that rises from the resurgence of neoliberalism and within the framework of structural changes in the field of technology that give rise to new forms of production and distribution of audiovisual material. The political and activist actions of the representative associations of the sector -the majority constituted between the end of the 9os and the beginning of the 2000's- translated in the period approached in a recognition and special treatment by the National Institute of Cinema and Audiovisual Arts for documentary production, generating a promotion and subsidy strategy. In the first place, we focus on some peculiarities of the Argentine film industry, to explain the high economic and geographical concentration that characterizes it and the imperative need for state support to sustain the cinematographic activity over time. In the second place, this paper researches the policies sustained in the last decades by the Argentine State in the cinematographic sector, which have marked a gap between production and commercialization. In the third place, the changes produced in the Argentine and

\section{Keywords:}

cultural policies, documentary films 
Latin American film industry are analyzed from the year 2008 onwards with the advance of digitalization, which although it represents an immediate improvement in the exhibition conditions, it has medium and long term consequences that accentuate the oligopolistic concentration. Fourthly, the field of documentary film production is addressed, giving an account of the forms of work and the circuits of exhibition of the groups of filmmakers, which are consolidated in the framework of the Argentine crisis of $200 \mathrm{r}$ and the changes that digital technology brings about. In the fifth place, the progress made in the period is examined according to the demands of the associations of documentary film makers through the Promotion Plan of the National Institute of Cinema and Audiovisual Arts, as well as the unresolved issues regarding the distribution and exhibition of Documentary films.

\section{Resumo}

\section{As políticas culturais na indústria cinematográfica: a produção e exibição de cinema documentário na Argentina}

O presente trabalho visa dar conta da política cultural promovida em nosso país no início do século XXI em relação à indústria cinematográfica. É interessante investigar uma área geralmente subestimada no orçamento do Estado, ou pelo menos náo levada em conta no mesmo nível da produção, circulaçáo e difusão de filmes de ficção: o campo do cinema documentário. É um campo que se torna relevante na Argentina desde 200I, quando a produção de alguns cineastas que vinham se desenvolvendo na transição democrática, soma-se à de muitos grupos de cineastas no contexto de crise política, social e econômica que é gestado com o ressurgimento do neoliberalismo e no quadro de mudanças estruturais no campo da tecnologia que dáo origem a novas formas

Palavras-chave: políticas culturais, documentários 
de produção e distribuição de material audiovisual. As associaçôes políticas e militantes que representam a indústria - a maioria formada entre o final dos anos 90 e início dos anos 2000- resultará no período coberto pelo reconhecimento e tratamento especial por parte do Instituto Nacional de Cinema e Artes Audiovisuais para produção documental, gerando uma estratégia de promoção e subsídio. Em primeiro lugar, há algumas peculiaridades da indústria cinematográfica argentina, para explicar a alta concentração econômica e geográfica que a caracteriza e a necessidade imperativa de apoio do Estado para sustentar a atividade cinematográfica ao longo do tempo. Em segundo lugar, investiga as políticas sustentadas nas últimas décadas pelo Estado argentino no setor cinematográfico, que marcaram uma lacuna entre produção e comercialização. Em terceiro lugar, as mudanças produzidas na indústria cinematográfica argentina e latino-americana são analisadas a partir de 2008 com o avanço da digitalizaçáo, que apesar de representar uma melhoria imediata nas condiçóes de exibição, tem consequências de médio e longo prazo que acentuam a concentração oligopolista. Quarto, o campo da produção de documentários é abordado, dando conta das formas de trabalho e dos circuitos de exibição dos grupos de cineastas, que se consolidam no marco da crise argentina de20or e as mudanças que a tecnologia digital. Quinto, o progresso realizado no período é examinado de acordo com as demandas das associaçóes de documentalistas através do Plano de Promoção do Instituto Nacional de Cinema e Artes Audiovisuais, bem como das contas pendentes, especialmente quanto à distribuição e exibição do cinema documentário. 


\section{La industria cinematográfica argentina: una alta concentración económica y geográfica}

$\mathrm{Al}$ analizar el mercado exterior de Argentina en el período 2002-2008, Calcagno (2009) señala la presencia constante que mantiene el sector audiovisual dentro del volumen de bienes característicos ${ }^{1}$ exportados y la relevancia que adquieren en ese contexto los servicios de productoras cinematográficas argentinas con una tendencia al crecimiento sostenido. La producción fílmica argentina ha ido en aumento a partir de la aplicación de la Ley de Cine (1995): en el período I995-I999 se registra un promedio de 33 largometrajes anuales; entre 2000 y 2005 , 60 largometrajes por año, subiendo a Ioo películas entre 2005 y 2010 (González, 20I4). Estos números dan cuenta del dinamismo que el sector cinematográfico argentino ha mostrado en las últimas décadas, y a ello habría que sumar lo producido en el interior del país, particularmente documentales no registrados en el Instituto Nacional de Cine y Artes Audiovisuales (en adelante INCAA). Sin embargo, diversos autores coinciden en plantear la imperiosa necesidad de apoyo estatal para sostener la actividad cinematográfica en el tiempo (González, 20I4; Campero, 2009; Schmoller, 2009; Rovito y Raffo, 2003). En mayor o menor medida, en prácticamente todos los países, si el Estado no aporta dinero ni impone restricciones a los exhibidores, no pueden realizarse ni exhibirse filmes nacionales en gran cantidad. Particularmente en nuestro país, se trata de una actividad subsidiada por la inversión pública estatal en un alto porcentaje:

La totalidad de la producción requiere del apoyo del Instituto Nacional de Cine y Artes Audiovisuales, que mediante créditos a la producción y subsidios a la exhibición, financia alrededor del $75 \%$ del costo de los largometrajes nacionales ( $\$ 1,5$ millones promedio), incrementando ese porcentaje casi al Ioo \% en el caso del documental. (Becerra, Hernández y Postolski, 2003:69-70)

En ello inciden fundamentalmente dos factores: la concentración económicageográfica de la producción y la condensación de la distribución y exhibición en grandes empresas oligopólicas. Hacia fines del siglo XX, con la liberalización

1 Son considerados bienes característicos aquellos cuyo valor está definido por su contenido intangible o simbólico (como un libro, un disco o una película), a diferencia de los productos auxiliares (insumos y maquinaria) y los productos conexos (los que permiten reproducir la imagen y el sonido). Si bien los bienes auxiliares y conexos no constituyen en sí mismos bienes culturales, en el análisis de Calcagno han sido considerados parte del comercio exterior cultural porque son imprescindibles para la producción de los bienes característicos (Calcagno, 2009). 
de los mercados —impulsada en América Latina a través de políticas neoliberales- aquellas industrias culturales que aún conservaban su raigambre nacional cambian totalmente su carácter a partir de la fusión de capitales e intereses en empresas globales. Así lo explica Néstor García Canclini:

Las industrias culturales continúan produciendo libros, discos, películas y programas televisivos, pero con la tendencia a seleccionar y espectacularizar aquellos "productos» que pueden competir con los de otras regiones y triunfar en el mercado. $\mathrm{Y}$ los mercados comunes no acaban de constituirse, según se ve en el Mercosur, o cuando más o menos lo logran, como en el Tratado de Libre Comercio de América del Norte, hacen como si solo se tratara de liberalizar los flujos comerciales, sin considerar qué le pasa al resto de la economía. (2002:31)

Estos conglomerados empresariales cuentan con las condiciones necesarias para incorporar las más recientes tecnologías de la información y la comunicación y reorganizar la producción de bienes culturales en función de una nueva división del trabajo, donde las acciones o partes del proceso están encadenadas más allá de su ubicación espacial. Incluso en aquellos países en donde el negocio del cine se ha vuelto altamente rentable (como Estados Unidos, China o India), el sector cinema- tográfico se beneficia de subsidios directos y de diversas estrategias que favorecen a las empresas de la industria (desgravaciones y exenciones fiscales, pagos diferidos, amortizaciones aceleradas). Las grandes productoras norteamericanas recuperan el costo de producción de sus películas en el mercado interno y, descontando el costo de la publicidad y las copias, todo ingreso generado en el mercado externo es ganancia neta. De la totalidad de las películas producidas, solo unas pocas se convierten en éxitos comerciales altamente redituables. Por otra parte, la inversión a realizar aumenta considerablemente cuando se suma el costo de las campañas de difusión multimediática orientadas a generar rápidamente un volumen significativo de espectadores que permita que el filme continúe en cartelera semana a semana (Rovito y Raffo, 2003).

En este contexto, las grandes empresas de Hollywood — las denominadas majors (Buena Vista, UIP, Warner, Fox, Sony) son las únicas que pueden ofrecer mayor cantidad de títulos y aquellos estrenos más comerciales, así como las que poseen mayor capacidad de presión sobre las salas de exhibición. En Argentina, a principios del siglo XXI, entre el $40 \%$ y $50 \%$ de las salas comerciales — complejos con cuatro o más pantallas- estaban manejadas por tres multinacionales extranjeras (Hoyts, Cinemark y National Amusements) (González, 20I4). En ese entonces, solo dos empresas nacionales 
tenían una porción significativa del mercado total: Atlas y Cinemacenter, con entre el $6 \%$ y el $7 \%$ promedio, respectivamente (Rovito y Raffo, 2003). En detrimento de las salas de pantalla única, en nuestra región se han expandido en las últimas décadas los complejos multisala, altamente equipados y ubicados en centros urbanos y lugares de alto poder adquisitivo (shoppings). Según la encuesta de consumos culturales y entorno digital realizada en el año 2013, la gran mayoría de los argentinos concurre a estos complejos y solo un $5 \%$ al cine de sala única; comportamiento que no exhibe diferencias según sexo ni nivel socioeconómico (Ministerio de Cultura de la Presidencia de la Nación. Sistema de Información Cultural de la Argentina, 20I3). Tras el cierre de gran número de salas de exhibición entre 1985 y 1994, actualmente solo unas pocas provincias tienen un mercado cinematográfico relevante (Buenos Aires, Santa Fe, Córdoba, Neuquén y Mendoza), siendo los grandes centros urbanos y las zonas más ricas del país los espacios que cuentan con salas de exhibición (Secretaría de Cultura de la Presidencia de la Nación. Sistema de Información Cultural de la Argentina, 20Io).

A la concentración económica que caracteriza a la industria del cine en general, se suma en nuestro país la concentración geográfica: los filmes se producen y circulan fundamentalmente en Buenos Aires y Gran Buenos Aires.
Ésta es una peculiaridad que se replica en la totalidad de las industrias culturales, acentuando las desigualdades hacia el interior del territorio argentino (Villarino y Bercovich, 20I4). Un aspecto a revisar en relación a políticas culturales, y que sin duda contribuiría a amenguar las desigualdades existentes, es la circulación de la producción cinematográfica en la región latinoamericana:

si en América Latina existiera una circulación cinematográfca regional óptimamente eficiente, los argentinos — que en promedio concurren una vez al cine al añopodrían tener en teoría más de 300 filmes regionales anuales entre los cuales escoger. Sin embargo, ello no ocurre: anualmente se estrenan entre 3 y io filmes latinoamericanos no nacionales — cifra similar a la que se da en los otros países de la regióncuyo público representa, en promedio, el $0,2 \%$ del total de los espectadores de cine. (González, 20I4:6IO)

Ciertas medidas tendientes a mejorar las condiciones de exhibición y circulación de producciones fílmicas a nivel regional se hallan en la actualidad escasamente desarrolladas y han tenido todavía poca repercusión. El acuerdo firmado en 2008 con la Unión Europea en la Reunión Especializada de Autoridades del Cine y el Audiovisual del Mercosur (RECAM) se caracteriza por la lentitud en su implementación. A través del Programa 
Mercosur Audiovisual (PMA) se prevee la construcción de una red de 30 salas digitales en Argentina, Brasil, Paraguay y Uruguay destinadas a la exhibición exclusiva de filmes de la región, pero al momento poco se ha avanzado en esta dirección (González, 20I4).

\section{Políticas culturales en la industria cinematográfica argentina: desfasaje entre producción comercialización}

Las políticas públicas en materia de cine implementadas en la primera década del siglo XXI se han enfocado en el plano de la producción y los aspectos vinculados a la distribución y exhibición han permanecido desatendidos por las legislaciones y medidas estatales (González, 20I4; Kamin, 20II; Schmoller, 2009). Algunos números nos permiten dimensionar los efectos de este desfasaje:

en los últimos tres años las mayores recaudaciones se concentraron en unas pocas películas mientras la gran mayoría de ellas solo fueron vistas por muy pocos espectadores. En 201 con IO2 películas estrenadas solo tres recaudaron el $75 \%$ de las entradas. En 2012 con 132 películas estrenadas solo cuatro recaudaron el $82 \%$ de las entradas.
En 2013 con 155 películas estrenadas solo cinco recaudaron el $89 \%$ de las entradas. (Kamin, 20II:s/n)

Desde mediados de la década del '9o la producción de filmes en Argentina tiende a aumentar, ${ }^{2}$ pero el promedio de espectadores por filme es uno de los más bajos entre los ocho mercados latinoamericanos más grandes y con mayor producción (en orden de escala Brasil, Colombia, México, Venezuela, Chile, Perú, Argentina y Uruguay) (González, 20I4). En ello ha incidido sin duda el aumento del boleto cinematográfico - que se cuadriplicó en la última década, sobre todo a partir de 2009 cuando se impuso el cine digital en $3 \mathrm{D}$ (González, 20I4) y una tendencia que viene creciendo a nivel mundial: la oferta de exhibición de películas por diferentes medios y formatos (televisión abierta, de pago, por cable, a través de plataformas digitales, video-home) (Sartora, 2009:66). Sin embargo, al analizar las estadísticas en el caso del cine, la cantidad de entradas vendidas es un dato que debe ser tomado con precaución. Debe ser analizado a partir de un hecho incontrastable: en la actualidad ya no existe un público de cine homogéneo y masivo, o existe para

2 Esta tendencia solo es detenida a partir de 2009 por la crisis económica internacional, que afecta a España, país con el cual se habían realizado numerosas coproducciones (Schmoller, 2009). 
muy pocas películas, como las producciones destinadas al público infantil o los estrenos comerciales a nivel mundial provenientes de la industria hollywoodense (Campero, 2009). La fragmentación del público es un aspecto relevante que sin lugar a dudas merece atención por parte de las políticas culturales.

En el período analizado se encuentra en vigencia una serie de resoluciones y medidas orientadas a regular desde el Estado la exhibición y duración de las películas argentinas en cartel. A través de la Resolución $\mathrm{N}^{\circ}$ 20I6/04, el INCAA amplía el concepto de cuota de pantalla (la cantidad mínima de películas nacionales que deben exhibir obligatoriamente las empresas exhibidoras), establece una clasificación de películas argentinas según la cantidad de copias con que se estrenan y crea un circuito de exhibición alternativo garantizando mediante acuerdos con las empresas exhibidoras la permanencia mínima en dos semanas de aquellas películas que se estrenan con un número de copias reducido 3 (Secretaría de Cultura de la Presidencia de la Nación, 2010). La cadena de exhibición oficial, conocida como los Espacios INCAA, abarca prácticamente la totalidad del territorio argentino y busca garantizar la difusión de producciones cinematográficas argen- tinas, incluidas las de estreno comercial, formato digital y cortometrajes. Esta medida ha contribuido a la expansión del sector cinematográfico, sobre todo para aquellas localidades que luego de la década del '9o habían quedado sin cines: el precio es accesible en comparación con el costo de las entradas en complejos multipantalla y el público se beneficia con descuentos generados a partir de convenios entre el INCAA y diversos sindicatos, gremios y asociaciones. No obstante, considerada en el marco de la industria cinematográfica, estas medidas no han transformado el panorama general del mercado: las recaudaciones y niveles de convocatoria de estos espacios son exiguos en comparación con las otras ofertas de exhibición (Kamin, 20II). Por ello es considerada un buen punto de partida, pero solo eso: punto de partida que necesariamente debe ser fortalecido y acompañado de otras estrategias.

La implementación de la cuota de pantalla en realidad no es efectiva si no se regula al mismo tiempo la cantidad de copias de los estrenos de la industria hollywoodense. Estas empresas oligopólicas distribuyen sus productos cinematográficos en condiciones de dumping, lo cual desvirtúa el Acuerdo General sobre Aranceles Aduaneros y el Comercio (GATT) y causa un perjuicio a

3 A través de la Resolución № 26/09 se introduce en la clasificación de películas argentinas una modificación en la cantidad de copias con que se estrenan (categoría a con 35 copias o más, categoría b entre 6 y 34 copias, categoría c hasta 5 copias). 
la producción argentina (Rovito y Raffo, 2003). En nuestro país no se cobra un impuesto significativo a la importación de largometrajes norteamericanos, mientras que la importación de película virgen para filmar en la Argentina debe pagar un impuesto proporcional a su costo y las sociedades de capital argentinas que producen cine deben tributar el $35 \%$ de su ganancia neta imponible. Las empresas de cine de Estados Unidos venden sus productos a un precio menor al costo de producción, haciendo la diferencia a partir de las entradas vendidas en el mercado interno y de los derechos de venta para DVD y televisión. Ello es posible por la concentración conglomeral de la industria cinematográfica norteamericana y por el estreno simultáneo a escala global que simplifica la campaña publicitaria (Campero, 2009).

Así, al momento de su presentación pública, las películas argentinas están siempre en desventaja. Kamin (20II) nos ofrece algunos argumentos para explicar el porqué de esta situación:

Una película nacional debe competir, al momento de su estreno, con otros productos cuyo poder de difusión es notablemente superior. Los costos de lanzamiento son de una muy alta exigencia económica que el productor nacional no está en condiciones de afrontar excepto en los casos en que reciba el apoyo de los grandes medios y/o acuerde con distribuidoras internacionales el gasto en las campañas de difusión y publicidad.
A pesar de las medidas orientadas a descentralizar la comercialización, podemos afirmar que en el período analizado las condiciones de acceso a la exhibición y distribución de filmes en nuestro país siguen dependiendo de las grandes compañías ligadas a Hollywood. Las disposiciones no parecen cumplirse con rigor y muchos de los empresarios de las salas multipantalla prefieren abonar las multas en vez de sacrificar los ingresos por los estrenos de filmes provenientes de la industria hollywoodense (Kamin, 20II).

Para resolver los problemas de la industria cinematográfica vinculados a la exhibición, el Estado, además de imponer cuota de pantalla o media de continuidad a todas las salas cinematográficas (incluyendo los multicines), debiera ocuparse de promover circuitos de cine alternativos, que no se guíen exclusivamente por la lógica de mercado. La interacción entre la industria cinematográfica y televisiva es sin duda una de las cuentas pendientes para fortalecer la difusión de la producción cinematográfica nacional. El artículo 67 de la Ley de Servicios de Comunicación Audiovisual, promulgada bajo el gobierno de Cristina Fernández de Kirchner en 2009, constituye una buena base para avanzar en ese sentido. En el Cap. V, referido a contenidos de la programación, la ley obliga a los canales de aire a adquirir con dinero los derechos de emisión de las películas argentinas (Ley 26522, 2010:38), siendo que 
convencionalmente estos derechos eran obtenidos a través de canjes por segundos de publicidad. Sin embargo, al momento la medida ha tenido poca repercusión, siendo contados los casos en que fue respetada y cumplida. Debería buscarse la forma para que la televisión asuma un rol más activo, que vaya más allá del pago de impuestos y se abran así más espacios en canales privados de aire y estatales para la difusión de cine argentino. Si bien dicha ley no fija un precio básico en la compra de derechos de antena ni identifica con claridad a los beneficiarios activos de las cuotas de pantalla, abre la posibilidad de una mayor interacción de los canales televisivos con el cine.

\section{La digitalización en el cine: ¿cambios en la concentración oligopólica?}

El avance en la digitalización de las salas cinematográficas impulsada en toda América Latina a partir de 2008, si bien trajo aparejado en lo inmediato una mejora en las condiciones de exhibición, tiene a mediano y largo plazo consecuencias que acentúan la concentración oligopólica. La digitalización supone reemplazar los proyectores analógicos por proyectores digitales y un cambio en los métodos de distribución de las películas, ${ }^{4}$ abriendo la posibilidad de proyección estereoscópica $(3 \mathrm{D})$. A partir de la digitalización todas las salas están en igualdad de condiciones para acceder a los títulos cinematográficos, modificándose el mercado de distribución de cine a nivel nacional con la posibilidad de realizar estrenos simultáneos: la distribución de contenidos - a través de satélite, internet y disco rígido- es más rápida, el proceso de proyección - a través del servidor de contenidos - se simplifica (con un solo un proyector en la cabina pueden exhibirse materiales $2 \mathrm{D}$ y $3 \mathrm{D}$ ) y la calidad del material se mantiene (no se produce desgaste por los sucesivos visionados) (Longobardi, 28 de julio de 2015).

En nuestro país, el convenio entre el INCAA, el Banco de Inversión y Comercio Exterior (BICE) y AR-SAT firmado en 2012 ha representado un avance en la transición digital: a través de este acuerdo el INCAA respalda a las empresas que encaran la compra de un proyector digital, es decir oficia de garante ante la entidad bancaria para agilizar las aprobaciones de

4 Los proyectores digitales se alimentan de servidores de contenidos: hay un software de gestión del servidor al que se vuelcan los contenidos encriptados. Las películas pueden usar discos duros como soporte o pueden ser descargadas directamente en los servidores de contenidos usando enlaces de banda ancha por satélite o por fibra. El tiempo que están los filmes en cartel y el número de visionados se controlan mediante claves generadas por la distribuidora, con un protocolo de control muy estricto. 
los préstamos, abarcando en primer lugar las salas pertenecientes al circuito establecido por el INCAA y en una segunda etapa las salas no regidas por la entidad. En 20I4, Lucrecia Cardoso, la entonces presidenta del INCAA, caracterizó la situación de los espacios de exhibición en Argentina:

Actualmente hay alrededor de 900 pantallas de cine en el país. De ese total aproximadamente el $60 \%$ está digitalizada y el otro $40 \%$ se encuentra dentro de los espacios que hay que acompañar mediante acciones como la constitución de este Fondo. ${ }^{5}$ (El INCAA aporta más fondos para la digitalización de la salas. Son aportes a un fondo de garantías para los que van a comprar los proyectores, I7 de abril de 20I4).

Sin embargo, desde una mirada de más largo plazo algunos autores observan en Argentina un estancamiento en el proceso de transición digital:

En 2009, Argentina era el tercer mercado latinoamericano con más salas digitales y para fines de 2012 era el quinto. Para septiembre de 2010 Argentina tenía el doble de salas digitales que Colombia, mientras que hacia marzo de 2013 el país caribeño tenía unas I2O salas digitales más que la Argentina. (González, s/f)

Según las estadísticas la digitalización se encontraba al 2014 en proceso de desarrollo, escasamente extendida en América Latina y ampliamente concentrada en unos pocos mercados:

en América Latina el promedio regional de digitalización es de $63 \%$ : sin embargo, si se quita a los mercados con mayor porcentaje de digitalización - México, Colombia y Centroamérica y el Caribe como un todo, con entre el $75 \%$ y el $80 \%$ de sus parques exhibidores digitalizados-, el porcentaje latinoamericano de digitalización caería al $44 \%$. A su vez, de las ocho mil salas digitales existentes en América Latina, el $67 \%$ se encuentran solo en dos países: México $(53 \%)$ y en Brasil (I4 \%). (González, 20I4:6I2-6I3)

En efecto, desde el comienzo de la transición digital México y Brasil concentran las tres cuartas partes del total de salas digitales latinoamericanas y las pantallas digitales en los países latinoamericanos se concentran en las ciudades capitales y en las zonas más prósperas. Brasil es el único caso en donde el Estado ha imple-

5 Se refiere al Fondo Fiduciario Específico para la Promoción y Estímulo de la Exhibición Cinematográfica, originado en el Convenio firmado en 2014 -en el marco de la creación de la Unidad de Digitalización y Nuevas Tecnologías Audiovisuales (Resolución N 1264/2014)-, que incluye acuerdos con los Bancos BICE y Nación, junto a la sociedad de garantías recíprocas Garantizar. 
mentado claramente acciones orientadas a apoyar el avance hacia la digitalización (ayudas financieras y exenciones impositivas, la conformación de un consorcio de exhibidoras nacionales para negociar con más fuerza el $\mathrm{VPF}^{6}$ con las majors y la instalación de integradores nítidamente establecidos en el mercado). A diferencia de países como Brasil y Uruguay, donde la importación y adquisición de proyectores y equipamiento digital no requiere el pago de tasa impositiva, en Argentina los exhibidores que se proponen digitalizar sus salas deben pagar cerca de un $50 \%$ más que en estos otros países (González, s/f).

Ciertos discursos que vinculaban el avance del cine digital a una democratización y diversificación en la industria cinematográfica, que en teoría sonaban muy promisorios, han encontrado en la práctica sus limitaciones y condicionantes: que en el «Primer Mundo»—: aunque este equipamiento redujo su precio en los últimos años, vale señalar que su mantenimiento es mucho más caro que el sistema de proyección en 35 milímetros, siendo la vida útil de los equipamientos digitales mucho menor (entre cinco y diez años) que la de los sistemas de proyección tradicional (unos 40 años). Así, la digitalización en los sistemas de proyección le genera un importante ahorro a las distribuidoras, pero no a las exhibidoras — aunque es cierto que gracias a la digitalización, las (escasas) salas de los interiores de los países ahora pueden recibir estrenos simultáneos, situación que antes era impensable (debían esperar varias semanas para recibir copias usadas, gastadas, de los estrenos que estaban en boca de todo el mundo, con grandes estrenos concentrados en las ciudades capitales y pocos centros urbanos más). (González, 20I4:6I5) si bien el cine digital reduce considerablemente los costos por copias, la instalación de estas salas digitales - que engloban proyector, periféricos, sistema operativo, equipamiento para exhibir en tercera dimensión y en algunos casos, hasta una pantalla distinta - ronda en América Latina los Ioo mil dólares cada una — casi el doble
De manera que, si bien trajo aparejado importantes beneficios — como el mejoramiento de las condiciones de calidad en la proyección, resolución de la imagen y la posibilidad de acceder a los estrenos-, al momento en Argentina la digitalización ha acentuado la concentración empresarial y geográfica,

6 La VPF (Virtual Print Fee) es una tarifa a la copia cinematográfica impuesta por las grandes distribuidoras transnacionales y los grupos de exhibición: su valor es abonado al realizarse cada visionado de la película y es próximo a lo que costaría una copia de 35 milímetros. En la práctica, esto ha perjudicado a los cines y distribuidoras pequeñas, que no logran compensar este costo con los ingresos percibidos en sala. 
debido fundamentalmente a sus costos de implementación y mantenimiento.

\section{El crecimiento del cine documental en la Argentina de 2001}

El cine documental ${ }^{7}$ cobra relevancia en Argentina a partir de 200I, en la coyuntura de crisis política, social y económica que se gesta a medida que el neoliberalismo muestra su cara más aguda y en el marco de cambios tecnológicos que dan lugar a nuevas formas de producción y distribución del material audiovisual: a la par que crecen los movimientos sociales de resistencia, se multiplica el número de realizadores y colectivos audiovisualistas.

Desde una formación político-militante, diversos realizadores venían destacándose en el campo del cine documental en los '80 y'9o (Carlos Echeverría, Marcelo Céspedes, Carmen Guarini, Tris- tán Bauer, Andrés Di Tella, Fernández Mouján entre otros), imprimiéndole a la producción una visión personal — de autor-, donde las fronteras entre documental y ficción aparecen como porosas. Paralelamente, a fines de los '8o y principios de los '9o se conforman colectivos de documentalistas que asumen el video —en ese momento analógico- como instrumento de intervención política para la organización y difusión de prácticas sociales de resistencia, dando origen a filmes de orientación no comercial, destinados a un público politizado, con productoras pequeńas, partidarias o independientes (Bustos, 2006; Dodaro, 2009). Estos grupos audiovisualistas pretenden registrar y denunciar la situación de desigualdad, resignificando la experiencia del cine militante de los '6o y'70 y articulando continuidades y rupturas en el campo cinematográfico. ${ }^{8}$ Algunos

7 Mucho se ha discutido en torno a la categoría cine documental, definido por oposición al cine de ficción y como una representación de la realidad, sobre todo porque se asocia generalmente a la imitación o mero registro del mundo histórico. Algunos autores recurren al término propuesto por el director y productor de cine norteamericano Frederick Wiseman para sus películas - ficciones de la realidad-para dar cuenta de los procesos de construcción y la subjetividad que incide en su realización (Weinrichter, 2004). Dado que es el término empleado en la legislación específica del campo cinematográfico y adoptado por las entidades de directores y productores, en este escrito se utiliza el término cine documental para hacer referencia a aquellas producciones cinematográficas que mantienen una relación indicativa con el mundo histórico, es decir, lo representan y sostienen una argumentación respecto al mismo (Nichols, 1997).

8 Dodaro (2009) caracteriza la labor de estos grupos como activismo audiovisual e invita a repensar la categoría de cine militante -tal como se gesta en nuestro país y otros de Latinoamérica en los años '60 y '70-, teniendo en cuenta los modos de apropiación de nuevas tecnologías, las diversas 
de estos colectivos se presentan como independientes, ${ }^{9}$ otros relacionados con movimientos políticos sin llegar a pertenecer a los mismos ${ }^{\text {Io }}$ y otros vinculados a partidos políticos de izquierda. ${ }^{\text {II }}$

La articulación entre los grupos sociales movilizados y los colectivos de arte y comunicación se refuerza en el contexto de privatización de los medios masivos de comunicación y formación de monopolios multimediales, que se gesta en los años'9o y obtura las posibilidades de enunciación. ${ }^{12}$ Con el surgimiento de Internet a finales de los '9o comienzan a elaborarse páginas web, a la vez que se incrementa la interconexión entre los grupos y los vínculos con redes y agencias de información alternativa (Dodaro, 2009).
Los cambios tecnológicos que suponen el pasaje del registro analógico a lo digital a principios del los años 2000 (unidades móviles más pequeñas y perfeccionadas, archivos digitales con gran capacidad de almacenamiento, la posprodución digital que posibilita mezclar gráficos, textos, audio y video) contribuyen a borrar los límites entre los perfiles profesionales (director, productor, camarógrafo, editor), favoreciendo nuevas formas de organización del trabajo. A diferencia de las productoras de cine comercial, en estos grupos los integrantes tienden a circular por las distintas tareas (cámara, producción y montaje o edición), y en la generalidad de los casos, las decisiones se adoptan de manera colectiva.

formas de entender la militancia y las articulaciones programáticas no partidarias de los realizadores, así como un contexto de libertad de expresión bajo una democracia formal.

9 El caso de Contraimagen, constituido en 1997, que opta por no hacer explícita su pertenencia a un partido político, aunque tiene de hecho un vínculo con el Partido de Trabajadores por el Socialismo (Bustos, 2006).

10 Pueden mencionarse: Alavío, cuya primer película data de 1993, que defiende la independencia partidaria y se relaciona con el Movimiento de Trabajadores Desocupados de Solano; Ojo Izquierdo, cuya fecha de origen no ha podido ser constatada pero por las fechas de sus producciones data de fines de los años ' 90 y principios de los 2000, vinculado al Movimiento de Trabajadores Desocupados de Neuquén; Adoquín video, conformado en 1988 o Cine Insurgente, constituido en 1998, que anhelan sumarse a alguna organización político-social (Bustos, 2006; Miranda, 2009; Repetto, 2009).

11 Como Ojo Obrero, constituido en 2001, que se presenta como el brazo audiovisual del Partido Obrero (Bustos, 2006).

12 En 1989, bajo el gobierno de Menem, se sanciona la Ley 23696, que elimina el artículo 45 de la Ley de Radiodifusión (22285/81), que prohibía a los dueños de medios gráficos ser propietarios de medios audiovisuales. Así se sientan las bases para la formación de grupos multimedia como Clarín, Atlántida o UNO América y la concentración se va a dar no solo en el ámbito privado. El Estado también va a concentrar medios: ATC, Canal 7, hoy TV pública, radio nacional, radios públicas (provinciales, municipales y universitarias). 
La tecnología digital abarata los costos de producción, así que a la vez que se multiplica la capacidad de producción, crecen las posibilidades de distribución y exhibición por canales alternativos a los comerciales. La exhibición es considerada por la mayoría de estos grupos una instancia de reflexión y debate, proyectando los filmes en primer lugar donde se registran las imágenes y teniendo a los actores de las películas - los participantes de movimientos sociales - como primeros espectadores (de la Puente y Russo, 2007). Algunos grupos han estrenado comercialmente sus filmes - los que contaron con financiamiento del INCAA, como el grupo Boedo Films, organizado desde 1992- o los han dado a conocer a través de festivales nacionales o internacionales, redes de cineclubes o eventos contraculturales. En general se conforman redes solidarias y horizontales de difusión, aunque hay que remarcar que en algunos grupos este tema no está tan discutido (Adoquín Video) y la circulación de las realizaciones es uno de los problemas de funcionamiento del colectivo (Ojo Obrero) (Bustos, 2006).

Con relación a las posturas tomadas por los grupos respecto del Estado, son variadas. Campo (2006) encuentra, básicamente, tres modos distintos de posicionamiento frente a las acciones del INCAA (subsidios y apoyos a sus realizaciones): voluntad de recibir un fomento o subsidio especifico para la actividad documental (Boedo Films); voluntad de recibir un subsidio pero, según lo dicho por sus integrantes, produciendo películas exclusivamente para «destruir al Estado» (Ojo Obrero); y negación absoluta a pedir o recibir cualquier tipo de apoyo del Estado (Cine Insurgente y Alavío). En caso de no contar o no solicitar subsidios, las producciones fílmicas se financian a partir de los aportes de los miembros del grupo o de las organizaciones sociales con las que trabajan, o con la venta de los materiales producidos (De la Puente y Russo, 2007).

El trabajo en red de los grupos de realizadores será clave durante 200I-2002, con Indymedia, ${ }^{13}$ Asociación de Docu-

13 Indymedia es un colectivo antiglobalización surgido en el marco de las protestas de Seattle (Canadá) en 1999. Representantes audiovisualistas de Argentina se suman en el año 2000, con acciones contra la Cumbre del ALCA. Funciona a partir de acuerdos que regulan el funcionamiento horizontal y prohibe la participación de partidos políticos 
mentalistas (AdoC), ${ }^{14}$ Argentina Arde, ${ }^{15}$ Kino Nuestra Lucha ${ }^{16}$ (Bustos, 2006). Además de préstamos de equipos, los grupos colaboran en la realización y circulación de materiales en crudo para diversas ediciones colectivas. El trabajo en conjunto los agrupamientos audiovisualistas en estos años fortalecen su capacidad de acción, adquiriendo una gran visibilidad además por el hecho de representar aquellas problemáticas que se han convertido en agenda para los medios de comunicación (Dodaro, Marino y Rodríguez, 20Io).

\section{Las asociaciones de} documentalistas como interlocutores ante el INCAA

Entre fines de los '9o y primeras décadas de los 2000 los colectivos de documentalistas y los realizadores independientes se agruparán en asociaciones, algunas de alcance nacional y otras de alcance regional, mancomunados en el fin de ejercer presión para que el INCAA reconozca un trato especial para la producción documental y se generen políticas estatales particulares para el sector. ${ }^{17}$ Las acciones de militancia audiovisual inciden en el

14 La Asociación de Documentalistas se crea en diciembre de 2001, al calor de la rebelión popular del 19 y 20, con más de 100 realizadores y productores. Funcionó hasta mediados de 2003 y adoptó la forma de un plenario abierto, donde las decisiones se votaban en asamblea y se informaban a través de boletines electrónicos. Los objetivos de AdoC se orientan a incidir sobre los marcos regulatorios del Estado (Dodaro, Marino y Rodriguez, 2010).

15 Inspirado en la experiencia político-artística Tucumán Arde, Argentina Arde se conforma a principios de 2002 y la Comisión Video cuenta con la participación de numerosos grupos (Cine Insurgente, Adoquín Video, Primero de Mayo, Boedo Films, Ojo Obrero, Contraimagen, Alavío, Indymedia, Adoc, Lenguas en los Pelos, Periodismo de Investigación Universidad Popular Madres de Plaza de Mayo, Escuela de Cine de Avellaneda y Estudiantes de Cine de la Universidad de La Plata).

16 Kino Nuestra Lucha se constituye en 2002, en el marco del $2^{\circ}$ Encuentro de Fábricas Recuperadas, con el objetivo de planificar tareas de difusión de dichas experiencias. Participan representantes de Contraimagen, Boedo Films, colectivo de arte y comunicación El Ojo Izquierdo de Neuquén y estudiantes de cine documental de la Universidad Popular Madres de Plaza de Mayo.

17 A Directores Argentinos Cinematográficos - Asociación General de Directores, Autores Cinematográficos y Audiovisuales (DAC, que data de 1958), se sumarán en este período otras de alcance nacional: Proyecto Cine Independiente (PCI, 1999), Documentalistas Argentinos (DOCA, 2007), Asociación de Realizadores Integrales, Directores, Productores y Técnicos de Cine Documental (RDI, 2008), Red Argentina de Documentalistas (RAD, 2010), Colectivo de Cineastas (CdD, 2016). Cabe mencionar otras, cuya fecha precisa de conformación no ha podido ser constatada, aunque por el registro de sus actividades se originan en la primera década del siglo XXI: Asociación Nacional de Directores y Productores de Cine Documental Independiente de la Argentina (ADN), Directores Independientes de Cine (DIC) y Asociación Argentina de Editores Audiovisuales (EDA). Con alcance 
plano normativo con una modificación en un artículo de la Ley Nacional de Cine (2004), que abre la posibilidad a los productores audiovisuales de obtener recursos materiales para la realización de documentales. Con la Resolución No 0658/04 se crea el Plan de Fomento del INCAA, incluyendo por primera vez el formato documental en la promoción estatal (cuarta vía: largometraje documental), aunque como en los casos de filmes de ficción el paso de exhibición debe ser de $35 \mathrm{~mm}$ o superior.

Si bien la Ley de Cine genera condiciones para la producción cinematográfica, no contempla en un primer momento las particularidades del cine documental. Los largometrajes considerados merecedores de subsidio por parte del INCAA a partir de esta ley son aquellos terminados en 35 $\mathrm{mm}$, un soporte no empleado usualmente para rodar y finalizar documentales por su alto costo y difícil recuperación. Recién en 2007 el INCAA empieza a tener en cuenta el cambio tecnológico que se venía produciendo: a través de la llamada vía digital o quinta vía documental se ofrecen subsidios más reducidos para aquellas películas filmadas y terminadas en digital (Resoluciones 632/o7/INCAA y 633/o7/INCAA). El hecho que esta vía no exija antecedentes por parte de los realizadores contribuye a la expresión de una gran diversidad temática y a la exploración estética y creativa.

Hacia 2007, representantes de Documentalistas de Argentina (en adelante DOCA) explicitaban cuáles eran sus

regional referenciadas en provincias pueden mencionarse: Asociación de Realizadores Audiovisuales de Neuquén (ARAN, 2001), Asociación Mendocina de Cine y Artes Audiovisuales (AMCAA, 2006), Red de Realizadores de Misiones - Asociación de productores cinematográficos y audiovisuales (2008), Asociación Cinematográfica de San Luis (ACSL, 2009), Asociación Civil de Realizadores de Santiago del Video (2009), Asociación Audiovisualistas del Chaco Argentino (ACHA, 2010), Asociación de Realizadores Audiovisuales de Santa Fe (ARAS, 2010), El Jaguar Asociación de Realizadores y Artistas Independientes de La Rioja (2010), Asociación Sanjuanina de Cine y Artes Audiovisuales (ASCAA, 2011), Asociación de Guionistas de San Luis (2011), Asociación de Productores Audiovisuales de Córdoba (APAC, 2011), Asociación de Realizadores Audiovisuales de Salta (ARAS, 2011), Asociación Civil Rionegrina de Artes Audiovisuales (ARNAA, 2011), Realizadores Audiovisuales de Formosa (RAF, 2011, bajo la figura de cooperativa), Asociación de Realizadores Audiovisuales La Rioja (ARA, 2014), Asociación de Realizadores Audiovisuales Multimediales de Misiones (ARAMMIS, 2015), Tucumán Audiovisual Asociación de Trabajadores Audiovisuales de Tucumán (2016), Asociación Jujeña de Realizadores Audiovisuales (AJRA, 2016). Cabe mencionar otras, cuya fecha precisa de conformación no ha podido ser constatada, aunque por el registro de sus actividades se originan en la primera década del siglo XXI: Asociación de Realizadores Audiovisuales de Entre Ríos (ARAER), Asociación Correntina de Realizadores Audiovisuales (ACRA), Asociación de Realizadores Audiovisuales de Chubut (ARACH), Asociación de Realizadores Independientes de Catamarca (ARIC). 
demandas ante el INCAA: aumento en el subsidio de cine documental, cambios en el modo y los tiempos de otorgamiento de subsidios, revisión en los criterios de conformación de los jurados que evalúan los proyectos fílmicos, subsidios para espacios alternativos de proyección (Minaso, 2007). En 2009, gran parte de los realizadores plantea la necesidad que el INCAA revise los criterios de selección de los filmes a subsidiar y logre un equilibrio entre todas las propuestas, incluyendo óperas primas, documentales y películas realizadas en digital (Schmoller, 2009). Ante esta situación, sin duda el Plan de Fomento del año 20II (Resoluciones $\mathrm{N}^{\circ}$ 2202/II/INCAA, I023/II/INCAA y I347/II/INCAA) marca un antes y un después en el ámbito de la producción documental: ${ }^{18}$ contempla las diversas instancias para el desarrollo de proyectos, subsidios y/o créditos a la producción y edición en DVD, a la vez que dictamina la conformación de los jurados que evalúan los proyectos con representantes de las entidades del sector documental. ${ }^{19}$

En las Jornadas de Reflexión y Debate para el Cine Documental —realizadas en la Escuela Nacional de Experimentación y Realización Cinematográfica (ENERC) en diciembre de 2014 y mayo de $2015-$ representantes de diversas asociaciones del campo cinematográfico (DOCA, Directores Argentinos Cinematográficos —en adelante DAC— Asociación Nacional de Directores y Productores de Cine Documental Independiente de la Argentina - $\mathrm{ADN}$ - y Asociación Argentina de Editores Audiovisuales EDA-) solicitan la incorporación formal de la vía digital en el Plan de Fomento, el aumento de la vía digital según el io \% del costo medio de película nacional y la independencia política de sus jurados, el establecimiento de políticas públicas de exhibición y difusión con una vía de apoyo al lanzamiento de los largometrajes (no solo para aquellos producidos con apoyo del INCAA, sino también para los proyectos independientes) y la implementación de ciertos mecanismos para avanzar en la federalización de la producción por la vía digital, con jurados elegidos en asambleas regionales abiertas y democráticas (Jornadas DOCA de reflexión y propuestas para el cine documental, 20I5).

En el documento elaborado por seis asociaciones nacionales de cine documental

18 Este Plan ha sido consensuado con algunas de las asociaciones que nuclean a los realizadores: Asociación de Realizadores Integrales de Cine Documental (RDI), Documentalistas de Argentina (DOCA) y Asociación Nacional de Directores y Productores de Cine Documental Independiente de la Argentina (ADN).

19 Este Plan de Fomento introduce las modificatorias de la Resolución 1880/08/INCAA (Resoluciones No 156/09/INCAA, 2735/09/INCAA, 161/10/INCAA, 196/10/INCAA, 2057/10/INCAA). 
de Argentina (ADN, DAC, DIC, DOCA, PCI, RDI) ${ }^{2 \circ}$ (II de mayo de 20I6) se reconocen los avances logrados en materia de producción (comités de evaluación de proyectos rotativos y trimestrales designados a instancias de las asociaciones documentales nacionales reconocidas por el INCAA, diferentes líneas de subsidio, según se trate de documentales de alto, medio o bajo presupuesto), se sugiere implementar mecanismos para mejorarla (la creación de una cuarta línea de subsidio, que contemple la producción de series documentales) ${ }^{2 r}$ y abordar aquellos aspectos no tenidos en cuenta en relación a la exhibición (se propone un subsidio al lanzamiento para los documentales de las líneas A, B y C con el fin de fortalecer su estreno en sala cinematográfica, se requiere al INCAA la generación de segmentos específicos para el documental en los canales públicos y privados, una línea de ayuda específica para que los propios realizadores realicen la reedición de los documentales con el fin de adecuarlos a formatos televisivos, así como la continuidad y el mejoramiento del subsidio a la edición de DVD).

Las instancias más vulnerables para el documental son la exhibición y la distribución, que según los movimientos de documentalistas argentinos, no deben guiarse por los mismos criterios y parámetros que el cine comercial. Así lo expresan representantes de diversas asociaciones de productores y directores documentalistas:

Fernando Krichmar: -El tema es que hay una sola sala y muchas películas esperando estreno. Pedimos que haya varios Gaumones, que los Espacio INCAA estén gerenciados por gente que sepa de cine, que tenga creatividad. Capaz, en vez de estar toda la semana un documental, es mejor que esté dos días a la semana durante varios meses.

Fito Pochat: -El Gaumont tiene una lógica de programación como los cines comerciales y un documental no necesita dos, tres funciones diarias. Esa es una lógica que tiene el cine para producciones que buscan convocar gran cantidad de gente en poco tiempo. Para nuestro cine funciona el boca en boca y por eso necesitamos la permanencia, aunque con menos días y funciones. Vamos a estar alertas para no retroceder en la producción, porque no puede ser materia de cambio.

20 DIC corresponde a Directores Independientes de Cine, PCl corresponde a Proyecto Cine Independiente.

21 Para esta línea de financiación se especifican esquema de financiamiento, formatos a presentar, la consideración de antecedentes, requisitos para presentación, los porcentajes y los rubros de costos a cubrir en la financiación (Asociaciones nacionales de cine documental de Argentina ADNDAC-DIC-DOCA-PCI-RDI, 11 de mayo de 2016). 
Virna Molina: - Nosotros lo que estamos garantizando es la libertad del artista para poder generar una obra para su sociedad, para su país. Si empezamos a hablar de hacer un cine rentable, hablamos de la censura implícita. (García Moreno, 20I6).

Las políticas públicas orientadas a fortalecer la exhibición deben contemplar la especificidad del cine documental. Se requiere adoptar una lógica específica que permita la permanencia en el tiempo, la instalación en el público y el «boca a boca»: pocas funciones semanales durante muchos meses. La audiencia de cine documental es un público muy específico, por tanto la difusión debe ser direccionada - no masiva-y la proyección entendida como un evento, con la presencia del director, con debates o charlas.

Existen muy pocos espacios en las provincias, por fuera del circuito bonaerense, donde uno pueda concurrir semanalmente a ver cine documental. Cincuenta y dos de los cincuenta y tres Espacios INCAA son administrados por privados (excepto la sala Gaumont de la ciudad de Buenos Aires, que es propiedad del INCAA) y ante las demandas de los documentalistas, el INCAA plantea que no tiene injerencia en la administración de esos espacios y en la decisión de qué filmes proyectar. Sumamente valorable es la iniciativa de un grupo de directores de cine documental nucleados en DAC de difundir a través de una plataforma virtual un filme documental sin costo por mes. ${ }^{22}$ El fomento a la producción independiente para la exhibición en TV e Internet es una de las propuestas ofrecidas desde el INCAA ante las demandas de los documentalistas, pero las asociaciones nacionales las consideran instancias alternativas de exhibición, no políticas públicas de exhibición:

la salida al problema por la vía de la exhibición en TV e Internet, aparece como una iniciativa que pone de manifiesto, por un lado, cierto desconocimiento respecto de las narrativas y estéticas propias del lenguaje cinematográfico, que implican un modo de organización particular, diferente al lenguaje que utilizaríamos pensando en un formato televisivo o en una obra pensada para ser vista online. Si se fija desde el propio plan de fomento que las producciones cinematográficas independientes se estrenen exclusivamente en TV y tengan circulación por Internet, obligando de esta manera a pensar sus obras en función de un lenguaje y una forma de atención diferentes a los experimentados en el cine ¿̇no significaría esto la extinción del cine independiente como lenguaje? (Documentalistas de Argentina, 20I4:5) 
Dos propuestas deberían tenerse en hacer más operativa la circulación de cuenta para consolidar los circuitos de material audiovisual de registro docuexhibición de documentales: por un lado, mental. Pero ¿qué hay de «mirar cine en implementar subsidios para los gastos de el cine»? El principal camino que debe exhibición en los espacios ya existentes, recorrerse para avanzar en este sentido que se sostienen en forma particular o es sin duda el de diseñar e implementar privada (Minaso, 2007); por otro lado, ciertas estrategias para lograr la exhibiaprovechar la estructura de las univer- ción de filmes documentales en las salas sidades públicas nacionales, que cubren de todos los Espacios INCAA, de una la totalidad del territorio argentino, con manera sistemática y continua en el un criterio descentralizador, para montar tiempo. Ello exigiría una redefinición del en su infraestructura salas alternativas carácter y la autonomía de estos espacios de proyección (Campero, 2009; Wolf, a partir del aporte de todo el sector au2009). Ambas propuestas no demanda- diovisual, incluido el de los realizadores rían grandes gastos al Estado y podrían independientes.

Referencias bibliográficas

- APREA, G. (2008). Cine y políticas en Argentina. Continuidades y discontinuidades en 25 años de democracia. Buenos Aires: Biblioteca Nacional - Universidad de General Sarmiento.

- BATLLE, D. (2009) La era de la madurez. En Wolf, S (Ed.). Cine Argentino. Estéticas de la producción (pp. 55-77). Ciudad Autónoma de Buenos Aires: BAFICI - Ministerio de Cultura, Gobierno de la ciudad de Buenos Aires.

- BECERRA, M.; HERNÁNDEZ, P. Y POSTOLSKI, G. (2003). La concentración de las industrias culturales. En Schargorodsky, H. (Comp.). Industrias culturales: mercado y políticas públicas en Argentina (pp. 55-84). Ciudad Autónoma de Buenos Aires: Ediciones Ciccus - Secretaría de Cultura de la Nación.

- BORÓN, A. (1995). El experimento neoliberal de Carlos Saúl Menem. En VV. AA. Peronismo y menemismo. Buenos Aires: El cielo por asalto.

- BUSTOS, G. (2006). Audiovisuales de combate. Acerca del videoactivismo contemporáneo. Buenos Aires: La Crujía. 
- CALCAGNO, N. (2009). Límite tecnológico, expansión creativa. El comercio exterior de bienes y servicios culturales en Argentina. Recuperado de: http://www.untref.edu.ar/documentos/ indicadores_culturales/2009/Limite\%20-\%20Natalia\%20 Calcagno.pdf

- CAMPERO, A. (2009). Supongamos que existe una política cinematográfica. En Wolf, S. (Ed.). Cine Argentino. Estéticas de la producción (pp. 15-23). Ciudad Autónoma de Buenos Aires: BAFICI - Ministerio de Cultura, Gobierno de la ciudad de Buenos Aires.

- CAMPO, J. (2006). Extensión o Comunicación. Tesina para acceder al título de Licenciado en Ciencias de la Comunicación (orientación en Procesos Educativos). Facultad de Ciencias Sociales, Universidad de Buenos Aires.

- DE LA PUENTE, M. Y RUSSO, P. (2007). El compañero que lleva la cámara. Cine militante argentino. Buenos Aires: Tierra del Sur. - DODARO, C. (2009). El videoactivismo. Experiencias de resistencia cultural y política en la Argentina de los años noventa. Palabra Clave, 12(2), 235-244.

- DODARO, C.; MARINO, S. Y RODRÍGUEZ, M.G. (2010). Normalidad, excepción y oportunidades. Dinámicas cultural y política en el caso del activismo audiovisual (Argentina 2002-2004). Lavboratorio. Revista de Estudios sobre Cambio Estructural y Desigualdad Social, (23). Recuperado de https://publicaciones. sociales.uba.ar/index.php/lavboratorio/article/view/97/0

- ESCUDERO, M. (2009). Cuando la lucha social y el arte se toman de la mano. En Paladino, D. (Dir.). Documentalismo y militancia (2001-2008). Cuadernos de historia, crítica y teoría del cine. Instituto de Artes del Espectáculo. Facultad de Filosofía y Letras, Universidad de Bellas Artes.

- GARCíA CANCLINI, N. (2002). Latinoamericanos buscando un lugar en este siglo. Buenos Aires: Paidós.

-___ (1999). La globalización imaginada. Buenos Aires: Paidós. - GONZÁLEZ, R. (2014). La exhibición y la distribución de cine en Argentina, de espalda a la digitalizacion. En Abratte, L. (et al.) y Utrera, L.L. (Ed.). Actas IV Congreso de la Asociación Argentina de 
Estudios de Cine y Audiovisual (pp. 608-618). Ciudad Autónoma de Buenos Aires: AsAECA. Recuperado de: http://asaeca.org/ download/la-exhibicion-y-la-distribucion-de-cine-en-la-argentinade-espaldas-a-la-digitalizacion/

- GONZÁLEZ, R. (s/f). La digitalización de salas, a ritmo lento en América Latina. Otros cines. Recuperado de: http://www. otroscines.com/nota?idnota $=7256$

- KAMIN, B. (2011). Algunas reflexiones sobre el panorama actual del cine argentino. Recuperado de: otroscines.com/nota8182-algunas-reflexiones-sobre-el-panorama-actual-del-cine-a - MINISTERIO DE CULTURA DE LA PRESIDENCIA DE LA NACIÓN. Sistema de Información Cultural de la Argentina (2013). Encuesta de consumos culturales y entorno digital. Audiovisual. Cultura Argentina. Ciudad Autónoma de Buenos Aires: Ministerio de Cultura de la Presidencia de la Nación.

- MINASO, M. (2007). El documental es una razón de estado y no de mercado. Entrevista a Virna Molina y Ernesto Ardito. Recuperado de: http://arditodocumental.blogspot.com.ar/2007/10/ el-documental-es-una-razn-de-estado-y.html

- MIRANDA, S. (2009). Imágenes y sonidos para la militancia. En Paladino, D. (Dir.). Documentalismo y militancia (2001-2008). Cuadernos de historia, crítica y teoría del cine. Instituto de Artes del Espectáculo, Facultad de Filosofía y Letras, Universidad de Bellas Artes.

- MIRANDA, S. Y SORIA, C. (2009). Documentalismo de investigación. En Paladino, D. (Dir.). Documentalismo y militancia (2001-2008). Cuadernos de historia, crítica y teoría del cine. Instituto de Artes del Espectáculo, Facultad de Filosofía y Letras, Universidad de Bellas Artes.

- NICHOLS, B. (1997). La representación de la realidad. Cuestiones y conceptos sobre el documental. Barcelona: Paidós.

- REPETTO, J. (2009). Camino al andar. En Paladino, D. (Dir.). Documentalismo y militancia (2001-2008). Cuadernos de historia, crítica y teoría del cine. Instituto de Artes del Espectáculo, Facultad de Filosofía y Letras, Universidad de Bellas Artes. 
- ROVITO, P. Y RAFFO, J. (2003). El mercado y la política cinematográfica. En Schargorodsky, H. (Comp.). Industrias culturales: mercado y políticas públicas en Argentina. Ciudad Autónoma de Buenos Aires: Ediciones Ciccus - Secretaría de Cultura de la Nación.

- SCHMOLLER, E. (2009). Cuatro tiempos y un epílogo. En Wolf, S. (Ed.). Cine Argentino. Estéticas de la producción (pp. 25-36). Ciudad Autónoma de Buenos Aires: BAFICI - Ministerio de Cultura, Gobierno de la ciudad de Buenos Aires.

- SECRETARÍA DE CULTURA DE LA PRESIDENCIA DE LA NACIÓN. Sistema de Información Cultural de la Argentina (2010). Valor y símbolo. Dos siglos de industrias culturales en la Argentina. Buenos Aires: Secretaría de Cultura de la Presidencia de la Nación. - VILLARINO, J. Y BERCOVICH, F. (2014). Atlas Cultural de la Argentina. $1^{\circ}$ ed. Buenos Aires: Secretaría de Cultura de la Presidencia de la Nación.

-WEINRICHTER, A. (2004). Desvíos de lo real. El cine de no ficción. Madrid: T\&B Ed.

Artículos periodísticos y comunicados de prensa - ASOCIACIONES NACIONALES DE CINE DOCUMENTAL DE ARGENTINA ADN-DAC-DIC-DOCA-PCI-RDI (11 de mayo de 2016). Propuestas para el fomento del documental. Recuperado de: https:// www.facebook.com/ArgenDoc/posts/1075479889177634

- CABRERA, L. (26 de febrero de 2015). Cine documental independiente: de la sala a la calle. Recuperado de: http:// laura-cabrera.blogspot.com.ar/2015/02/cine-documentalindependiente-de-la.html

- DIRECTORES ARGENTINOS CINEMATOGRÁFICOS. Asociación general de directores, autores cinematográficos y audiovisuales (13 de abril de 2018). Formación de la mesa del cine y del contenido audiovisual nacional. Recuperado de: www.dacdirectoresdecine. org.ar/noticias/cdp2018-04-13-formacion-de-la-mesa-del-ciney-del-contenido-audiovisual-nacional

- DIRECTORES ARGENTINOS CINEMATOGRÁFICOS. Asociación general de directores, autores cinematográficos y audiovisuales (6 de junio de 2018). DAC no avala las medidas del INCAA Re- 
cuperado de: http://www.dacdirectoresdecine.org.ar/noticias/ cdp2018-06-06-dac-no-avala-las-medidas-del-incaa

- DOCUMENTALISTAS DE ARGENTINA (27 de abril 2018). Carta abierta a la comunidad audiovisual. Nos oponemos a la embestida contra el cine independiente. Recuperado de: https:// www.redeco.com.ar/nacional/gobierno/23845-carta-abierta-ala-comunidad-audiovisual

- DOCUMENTALISTAS DE ARGENTINA (5 de julio 2018). La lucha del cine documental. Recuperado de: https://www.facebook. $\mathrm{com} /$ notes/doca-documentalistas-de-argentina-/la-lucha-delcine-documental/1953165201420739/

- DOCUMENTALISTAS DE ARGENTINA (2014a). Hacia un nuevo Plan de Fomento. Pasado, presente, futuro del cine documental argentino. Las cifras y análisis de un cine en ascenso, sin espacio en las salas. Jornadas de reflexión y propuestas para el cine documental. Ciudad Autónoma de Buenos Aires.

- _ (2014b). Documento de Horco Molle. Por un cine documental independiente, descentralizado y federal. V Encuentro Nacional DOCA. Tucumán.

- El INCAA aporta más fondos para la digitalización de la salas. Son aportes a un fondo de garantías para los que van a comprar los proyectores (17 de abril de 2014). Cines Argentinos. Recuperado de: https://www.cinesargentinos.com.ar/noticia/4126el-incaa-aporta-mas-fondos-para-la-digitalizacion-de-la-salas/

- RED ARGENTINA DE DOCUMENTALISTAS (2015). Declaración de Mina Clavero. IV Encuentro Nacional de Documentalistas. Córdoba. - GARCÍA MORENO, M. (21 de mayo de 2016). El documental debe ser una verdadera política de Estado y no de mercado. Tiempo Argentino. Recuperado de: http://www.tiempoar.com. ar/articulo/view/57349/el-documental-debe-ser-una-verdaderapolitica-de-estado-y-no-de-mercado

- GIL, G. (12 de enero de 2003). Discurso de asunción al Ministerio de Cultura de Brasil. Página 12.

- JORNADAS DOCA de reflexión y propuestas para el cine documental (2015). Recuperado de: https://www.youtube.com/ watch?v=tIpC40bMPOI 
- LONGOBARDI, L. (28 de julio de 2015). Cines argentinos: digitalización y récord. Show.cero. Recuperado de: https://puntocero. $\mathrm{me} /$ cines-argentinos-digitalizacion-y-record/

- MINASO, M. (2007). El documental es una razón de estado y no de mercado. Entrevista a Virna Molina y Ernesto Ardito. Recuperado de: http://arditodocumental.blogspot.com.ar/2007/10/ el-documental-es-una-razn-de-estado-y.html

- YACCAR, M.D. (18 de mayo de 2018). Embestida contra el cine independiente. Página 12. Recuperado de: https://www.pagina12.com.ar/115498-embestida-contra-el-cine-independiente - DIGITALIZACIÓN Y NUEVAS TECNOLOGÍAS. Instituto Nacional de Cine y Artes Audiovisuales. Ministerio de Educación, Cultura, Ciencia y Tecnología de la Nación. Recuperado de: http://www. incaa.gov.ar/fomento-a-la-industria/promocion/digitalizacion-ynuevas-tecnologias

\section{Leyes y resoluciones}

- Ley 17741. Ministerio de Justicia y Derechos Humanos, Presidencia de la Nación. Buenos Aires, Argentina, mayo de 1968. Recuperada de: http://servicios.infoleg.gob.ar/infolegInternet/ anexos/15000-19999/17938/norma.htm

- Ley 23696. Honorable Congreso de la Nación Argentina. Buenos Aires, Argentina, agosto de 1989. Recuperada de: http:// servicios.infoleg.gob.ar/infolegInternet/verNorma.do?id=98

- Ley 26522. Reglamentación y Normas Complementarias. Honorable Cámara de Diputados de la Nación. Secretaría Parlamentaria. Dirección de Información Parlamentaria. Buenos Aires, Argentina, setiembre de 2010. Recuperada de: http://www1. hcdn.gov.ar/dependencias/dip/L\%2026522.pdf

- Ley 24377. Sala de Sesiones del Congreso Argentino. Buenos Aires, Argentina, setiembre de 1994. Recuperada de: http://servicios. infoleg.gob.ar/infolegInternet/anexos/0-4999/767/norma.htm - Resolución N²016/04, Instituto Nacional de Cine y Artes Audiovisuales, 2004. Recuperada de: servicios.infoleg.gob.ar/ infolegInternet/anexos/95000-99999/96178/norma.htm 
- Resolución N0658/04, Instituto Nacional de Cine y Artes Audiovisuales, 2009. Recuperada de: http://I.exam-10.com/ biolog/13181/index.html

- Resolución N 632/07, Instituto Nacional de Cine y Artes Audiovisuales, 2009. Recuperada de: blankspot.com.ar/prodav/ Res 632-07.pdf

- Resolución № 633/07, Instituto Nacional de Cine y Artes Audiovisuales, 2009. Recuperada de: http://servicios.infoleg.gob.ar/ infolegInternet/anexos/125000-129999/128035/norma.htm - Resolución N²6/09, Instituto Nacional de Cine y Artes Audiovisuales, 2009. Recuperada de: http://dev1.incaa.gob.ar/ wp-content/uploads/2016/04/RESOLUCION_CUOTA_DE_PANTALLA_26_2009.pdf

- Resolución N²202/11, Instituto Nacional de Cine y Artes Audiovisuales, 2011. Recuperada de: http://servicios.infoleg.gob. ar/infolegInternet/anexos/185000-189999/186667/norma.htm - Resolución N 1023/11, Instituto Nacional de Cine y Artes Audiovisuales, 2011. Recuperada de: http://servicios.infoleg. gob.ar/infolegInternet/anexos/180000-184999/181689/ norma.htm

- Resolución No 1347/11, Instituto Nacional de Cine y Artes Audiovisuales, 2011. Recuperada de: servicios.infoleg.gob.ar/ infolegInternet/anexos/180000-184999/182888/norma.htm - Decreto 267/15, Ente Nacional de Comunicaciones, 2015. Recuperado de: http://servicios.infoleg.gob.ar/infoleglnternet/ anexos/255000-259999/257461/norma.htm

- Decreto 13/15, Ley de Ministerios, 2015. Recuperado de: http://servicios.infoleg.gob.ar/infolegInternet/anexos/255000-259999/256606/norma.htm

- Decreto 324/17, Instituto Nacional de Cine y Artes Audiovisuales, 2011. Recuperado de: https://www.boletinoficial.gob.ar/\#! DetalleNorma/163295/20170509 